SUBJECT AREAS:

MOLECULAR

NEUROSCIENCE

SCHIZOPHRENIA

Received

20 September 2013

Accepted

2 December 2013

Published

18 December 2013

Correspondence and requests for materials should be addressed to

D.S. (duncans@mail.

med.upenn.edu)

\section{Dysregulation of glucocorticoid receptor co-factors FKBP5, BAG 1 and PTGES3 in prefrontal cortex in psychotic illness}

\author{
Duncan Sinclair ${ }^{1,2,3,4}$, Stu G. Fillman ${ }^{1,2,3}$, Maree J. Webster ${ }^{5}$ \& Cynthia Shannon Weickert ${ }^{1,2,3}$
}

${ }^{1}$ Schizophrenia Research Institute, Sydney, New South Wales, Australia, ${ }^{2}$ Neuroscience Research Australia, Sydney, New South Wales, Australia, ${ }^{3}$ School of Psychiatry, University of New South Wales, Sydney, New South Wales, Australia, ${ }^{4}$ Neuropsychiatric Signaling Program, Center for Neurobiology and Behavior, Department of Psychiatry, University of Pennsylvania, ${ }^{5}$ Stanley Medical Research Institute, Laboratory of Brain Research, 9800 Medical Center Drive, Rockville, Maryland 20850.

Molecular abnormalities within the glucocorticoid receptor (GR) stress signaling pathway may confer, or reflect, susceptibility to stress in schizophrenia and bipolar disorder, but the extent of such abnormalities in the brain is not known. Using RNA-Seq and qPCR in two postmortem cohorts totaling 55 schizophrenia, 34 bipolar disorder and 55 control individuals, we identified increased FKBP5 and PTGES3 mRNA expression, and decreased BAG1 mRNA expression, in the prefrontal cortex in schizophrenia cases relative to controls $(68.0 \%[p<0.001], 26.0 \%[p<0.01]$ and $12.1 \%$ [p $<0.05]$ respectively). We also observed increased FKBP5 and decreased BAG1 mRNA expression in bipolar disorder (47.5\% $[\mathrm{p}<0.05]$ and $14.9 \%[\mathrm{p}<0.005])$. There were no diagnostic differences in steady-state FKBP51 protein levels, nor in HSPA1A, HSP90AA1, DNAJB1 or HSPB1 mRNA levels. GR, co-factor and chaperone mRNA levels were strongly correlated. These results reveal coordinated cortical dysregulation of FKBP5, PTGES3, BAG1 and GR genes within the glucocorticoid signaling pathway in psychotic illness.

tress has long been considered a contributor to the onset, symptoms and disease course of psychiatric illness $^{1-8}$. In support of this hypothesis, dysregulation of hypothalamic-pituitary-adrenal axis activity, under basal conditions and during stress, has been observed in schizophrenia and bipolar disorder ${ }^{9-11}$. In both illnesses, decreased mRNA expression of the glucocorticoid receptor (GR), the primary receptor responsive to stress-induced cortisol secretion, has been observed using multiple post-mortem tissue cohorts and brain regions ${ }^{12-16}$. In prefrontal cortex of individuals with schizophrenia and bipolar disorder, increased expression of a truncated GR protein isoform has been reproducibly demonstrated ${ }^{14,15}$. It is not known whether these molecular stress signaling abnormalities in psychotic illness extend beyond GR, to include other cellular components such as stress receptor co-factors and chaperones which are integral to the stress response in the human brain.

Effective GR-mediated stress signaling relies on the coordinated activity of numerous co-factors and chaperones, which fold GR into a mature state, regulate its affinity for cortisol, facilitate its translocation into the nucleus, and modulate GR-mediated transcriptional activation or repression of target genes (Figure 1) ${ }^{17-21}$. Some of these co-factors and chaperones have been implicated in psychiatric illness. Single nucleotide polymorphisms (SNPs) within the gene for FKBP5, which binds GR and facilitates nuclear translocation, have been linked to bipolar disorder, major depression and post-traumatic stress disorder ${ }^{22-26}$, and have been associated with impaired cognitive performance in individuals with schizophrenia ${ }^{27}$. Increased FKBP5 gene expression has been reported in the frontal cortex in individuals with major depression ${ }^{28}$, while decreased FKBP5 mRNA and protein have been described in the amygdala in suicide completers ${ }^{29}$. Mood stabilizer treatments for bipolar disorder have been shown to up-regulate BAG1 expression ${ }^{30}$. Hsp70 (HSPA1A) and Hsp90 (HSP90B1) gene SNPs have been associated with schizophrenia and bipolar disorder respectively ${ }^{31-33}$. Increased mRNA expression of Hsp70 and Hsp27 has been observed in the prefrontal cortex of patients with schizophrenia ${ }^{34}$. Transcriptional changes in important stress chaperones suggest their involvement in psychotic illness, yet these findings have yet to be replicated, while a number of key molecular regulators of stress responsiveness have yet to be examined. Furthermore, since the ability of the brain to respond to stress depends on coordinated modulation of gene 


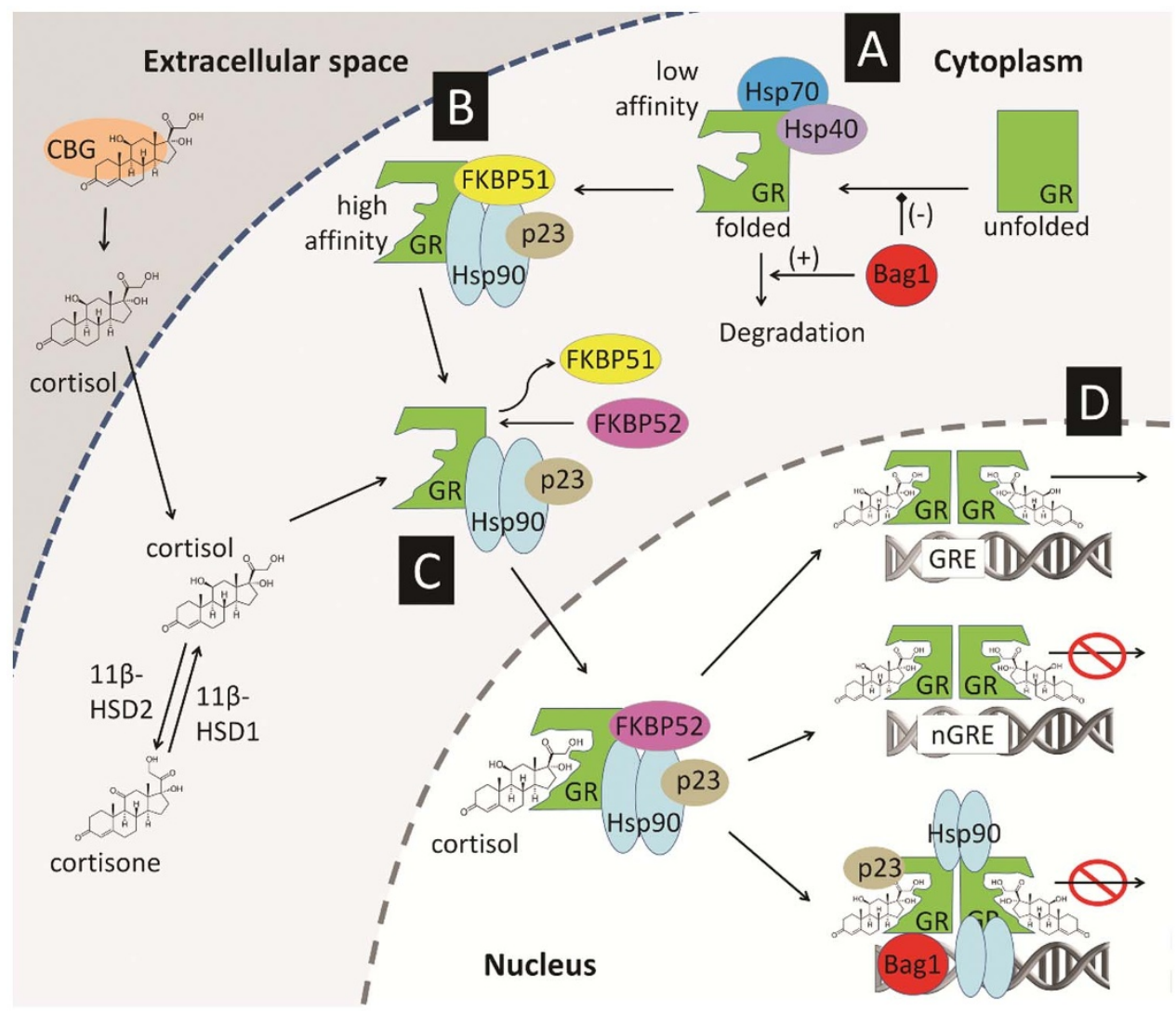

Figure $1 \mid$ The involvement of key co-factors and chaperones in GR-mediated stress signaling. (A) Hsp40 and Hsp70 facilitate, while Bag1 impairs, the folding of GR into a low steroid-affinity conformation. Bag1 also aids degradation of the unstable folded GR complex, (B) Hsp90, p23 and FKBP51 stablilise the GR complex in a high affinity state, (C) Displacement of FKBP51 by FKBP52 enables the translocation of the cortisol-bound GR heterocomplex to the nucleus, (D) Within the nucleus, GR activates or represses the transcription of target genes, at GREs or nGREs respectively. Overexpression of Bag1, Hsp90 or p23 can repress GR-responsive gene transcription. Availability of free cortisol (modulated by CBG) and interconversion of cortisol to cortisone by $11 \beta-\mathrm{HSD} 1 / 2$ also impact GR signaling. See Grad and Picard ${ }^{17}$ for comprehensive review. Abbreviations: GREglucocorticoid response element, nGRE- negative glucocorticoid response element, CBG- cortisol binding globulin, 11 $\beta$-HSD- 11 $\beta$-hydroxysteroid dehydrogenase.

expression, the relationships between expression of key stress chaperones and co-factors may illuminate how the GR stress pathway is regulated in cortical neurons to adapt to the demands of a changing environment.

In this study, we first used RNA sequencing in a cohort of 20 males with schizophrenia and matched controls to test if expression of stress receptor co-factors and chaperone genes FKBP5, FKBP4, PTGES3, BAG1, HSPA1A, HSP90AA1, DNAJB1 and HSPB1 was altered in the dorsolateral prefrontal cortex (DLPFC) of people with schizophrenia. These genes encode the FKBP51, FKBP52, p23, Bag1, Hsp70, Hsp90, Hsp40 and Hsp27 proteins respectively. The DLPFC brain region was employed because the most striking diagnostic differences in GR mRNA and protein expression in psychiatric illness have been evident in that region ${ }^{14-16}$. We then used qPCR, in an additional independent cohort of up to 35 schizophrenia cases, 34 bipolar disorder cases and 35 controls, to replicate these results and test if any observed changes in stress response-related mRNAs found in schizophrenia were shared with, or distinct in, individuals with bipolar disorder. Thereafter, informed by our mRNA findings, we focused on FKBP5, quantifying FKBP51 protein in schizophrenia and bipolar disorder and explored the possible effects of eight FKBP5 genetic variants on FKBP5 mRNA and FKBP51 protein in the DLPFC. We hypothesized that molecular stress signaling abnormalities in schizophrenia and bipolar disorder may extend beyond GR mRNA and protein to include key co-factors and chaperones, and that relationships between mRNA transcripts would reflect a pathway coordinately dysregulated, resulting in disruption in the fine-tuning of stress responsiveness in the human DLPFC.

\section{Results}

Stress signaling co-factor gene expression in the DLPFC in schizophrenia and bipolar disorder. Using RNA-Seq, amplified fragments mapping to mRNA transcripts for FKBP5, PTGES3, BAG1 and FKBP4 were quantified in the Sydney TRC cohort (for cohort details see Table 1). FKBP5 mRNA levels were significantly higher in schizophrenia cases than controls (61.5\%, ANCOVA F $(1$, $33)=8.93, p=0.005$; Figure $2 \mathrm{~A}$ ). The FKBP5 mRNA expression values of 10 (out of 20) schizophrenia cases were higher than the highest control FKBP5 expression value. These findings were not driven by effects of suicide in the schizophrenia group, because although FKBP5 mRNA levels varied significantly according to suicide status (ANCOVA $\mathrm{F}(2,32)=6.39, p<0.005$ ), FKBP5 expression levels within the schizophrenia (non-suicide) group were higher than controls ( $83 \%$ increase), whereas levels in the schizophrenia (suicide) group were not different to controls $(3 \%$ decrease). Levels of PTGES3 mRNA were significantly higher in schizophrenia cases relative to controls $(22.4 \%$, ANCOVA F $(1,33)$ $=7.27, p=0.01$; Figure $2 \mathrm{~B}$ ). There were no significant differences between schizophrenia cases and controls in the mRNA expression of BAG1 (ANCOVA F $(1,33)=0.35, p=0.56$ ) or FKBP4 (ANCOVA $\mathrm{F}(1,33)=1.00, p=0.33$; Figure $2 \mathrm{C}$ and $2 \mathrm{D})$. The achieved power of ANCOVA analyses ranged from 0.52 to 0.87 , and are provided in 
Table 1 | Demographics of schizophrenia and matched control subjects in the New South Wales Tissue Resource Center (TRC) post-mortem brain tissue cohort used in this study

\begin{tabular}{|c|c|c|}
\hline TRC cohort & Control group $(n=20)$ & Schizophrenia group $(n=20)$ \\
\hline Diagnostic subtype & - & $\begin{array}{l}\text { SZ(disorg.) }=3, S Z \text { (paranoid) }=11, S Z \text { (undiff.) }=5 \\
\quad S Z \text { (residual) }=1\end{array}$ \\
\hline Age in years (range) & $47.7(18-74)$ & $47.3(27-75)$ \\
\hline Gender & $20 M$ & $20 M$ \\
\hline Hemisphere & $11 R: 9 L$ & 7R:13L \\
\hline $\mathrm{pH} \pm \mathrm{SD}$ & $6.67 \pm 0.24$ & $6.63 \pm 0.24$ \\
\hline PMI (hours) \pm SD & $24.23 \pm 11.06$ & $29.59 \pm 16.11$ \\
\hline $\mathrm{RIN} \pm \mathrm{SD}$ & $7.36 \pm 0.39$ & $7.24 \pm 0.78$ \\
\hline Manner of death & $\begin{array}{l}\text { natural }=20 \\
\quad \text { (heart attack }=16, \text { other }=4 \text { ) }\end{array}$ & $\begin{array}{l}\text { natural }=15, \text { suicide }=5 \text { (heart attack }=12, \text { drug toxicity }=2, \\
\quad \text { hanging }=4, \text { CO Poisoning }=1, \text { other }=1)\end{array}$ \\
\hline Age (y) at onset (range) & - & $23.9(17-40)$ \\
\hline Illness duration $(y) \pm S D$ & - & $23.4 \pm 12.41$ \\
\hline $\begin{array}{l}\text { Lifetime antipsychotics } \\
\text { (Chlorpromazine equiv., mg) }\end{array}$ & & $594.94 \pm 280.11$ \\
\hline Antidepressant use & yes $=0$, no $=20$ & yes $=8$, no $=12$ \\
\hline Type of antidepressant* & - & $\mathrm{SSRI}=6, \mathrm{TCA}=2$ \\
\hline
\end{tabular}

Abbreviations: SZ-schizophrenia, M- male, L- left, R- right, SD- standard deviation, PMI- post-mortem interval, RIN- RNA integrity number, y-years. Data quoted are mean (range) + /- standard deviation.

Supplementary Table S1. Significant correlations of co-factor mRNAs with other clinical/demographic variables in the TRC cohort are described in Supplementary Results and Supplementary Table S1.

The number of normalized RNA-Seq reads mapping to specific FKBP5 exons in schizophrenia cases and controls were compared (Supplementary Results and Supplementary Table S2). Three FKBP5 exons which are common to FKBP5 mRNA transcripts 1-3, and one exon unique to transcript variant 4 , had significantly more mapped reads in schizophrenia cases than controls (all 1.4 to 1.9-fold increases, $0.005<p<0.05$ ).

These results were replicated by qPCR in the Stanley Array cohort (for cohort details see Table 2). FKBP5 mRNA levels (total expression of FKBP5 transcript variants 1-4) differed significantly according to diagnosis (ANCOVA F $(2,87)=6.00, p<0.005)$, with a $68.0 \%$ increase in schizophrenia cases relative to controls $(p<0.001$; Figure 2E). As in the TRC cohort, FKBP5 mRNA levels varied significantly according to suicide status (ANCOVA F $(4,85)=3.67$, p $<$ 0.01 ), with substantially higher FKBP5 expression levels within the schizophrenia (non-suicide) group than controls ( $87 \%$ increase), and marginally higher levels in the schizophrenia (suicide) group than controls (5\% increase). Increased FKBP5 mRNA expression was also seen in bipolar disorder cases relative to controls $(47.5 \%, p<0.05$; Figure 2E). No effects of suicide were seen in the bipolar disorder group. The FKBP5 mRNA expression values of 10 (out of 32) schizophrenia cases, and 6 (out of 29) bipolar disorder cases, were higher than the highest control FKBP5 expression value. PTGES3 mRNA expression was also $35.8 \%$ greater in schizophrenia cases than controls (Figure 2F). When testing whether findings from the TRC cohort were replicated, this increase was significant (one-tailed t-test, $p<0.01$ ), although the overall effect of diagnosis on PTGES3 expression did not reach significance (ANCOVA $\mathrm{F}(2,88)=2.70$, $p=0.07)$. Unlike in the TRC cohort, BAG1 mRNA levels differed significantly between diagnostic groups (ANCOVA $\mathrm{F}(2,91)=5.02$, $p<0.01$ ), with a $12.1 \%$ decrease in schizophrenia cases relative to controls $(p<0.05)$, and a $14.9 \%$ decrease in bipolar disorder cases relative to controls $(p<0.005$; Figure $2 \mathrm{G}$ ). There were no significant diagnostic differences in mRNA expression of FKBP4 (ANCOVA $\mathrm{F}(2,91)=2.11, p=0.13$; Figure $2 \mathrm{H})$. The achieved power of ANCOVA analyses, and significant correlations of co-factor mRNAs with other clinical/demographic variables in the Stanley Array cohort ranged from 0.74 to 0.83 , and are provided in Supplementary Table S1.
Stress signaling chaperone gene expression in the DLPFC in schizophrenia and bipolar disorder. When stress signaling chaperones were quantified by RNA-Seq in the Sydney TRC cohort, there were no significant differences between schizophrenia cases and controls in their mRNA expression levels: HSPA1A (ANCOVA F $(1,33)=$ $0.01, p=0.99$ ), HSP90AA1 (ANCOVA $\mathrm{F}(1,33)=2.85, p=0.10$ ), DNAJB1 (ANCOVA F $(1,33)=0.02, p=0.88$ ) or HSPB1 (ANCOVA $\mathrm{F}(1,33)=0.38, p=0.54$ ) (Figure 3A-D). Similarly, in the Stanley cohort, there were no significant differences in mRNA levels of HSPA1A (ANCOVA $F(2,89)=0.10, p=0.90$ ), HSP90AA1 $($ ANCOVA $\mathrm{F}(2,89)=0.69, p=0.50)$, DNAJB1 (ANCOVA F $(2$, $85)=0.11, p=0.89)$ or HSPB1 (ANCOVA $\mathrm{F}(2,90)=0.73, p=$ $0.48)$ between schizophrenia, bipolar disorder and control groups (Figure 3E-H).

Relationships between mRNA expression of GR transcripts, cofactors and chaperones in the DLPFC in the Stanley Array cohort. Spearman's correlations, using non-transformed data, were performed to explore the relationships between mRNA expression of GR transcripts, co-factors and chaperones in the DLPFC (Figure 4). Expression of total GR, GR-1B, GR-1C and GR-1H mRNA transcripts was previously quantified in the same cohort using the same $\mathrm{qPCR}$ methods ${ }^{16}$. The HSP chaperones and PTGES3 were significantly positively correlated with each other (all $0.29<\mathrm{r}<0.77, p<0.01$ ), as were co-factors BAG1 and FKBP4 $\left(\mathrm{r}=0.45, p<5 \times 10^{-6}\right)$. GR-1B mRNA was negatively correlated with FKBP5, PTGES3 and all HSPs (all $-0.19<\mathrm{r}<$ $\left.-0.53,1 \times 10^{-6}<p<0.08\right)$ and positively correlated with FKBP4 and BAG1 (all $0.23<\mathrm{r}<0.33, p<0.05$ ). FKBP5 was positively correlated with PTGES3 and all HSPs (all $0.20<\mathrm{r}<0.29, p<0.06$ ), and negatively correlated FKBP4 and BAG1 [trend-level significance: $r=-0.18(p=0.09)$ and $r=-0.19(p=0.06)$ respectively]. FKBP4 and BAG1 were not significantly correlated with any HSPs.

FKBP51 protein abundance in the DLPFC in schizophrenia and bipolar disorder. To determine whether FKBP5 mRNA alterations in schizophrenia and bipolar disorder (involving transcript variants 1-4) were accompanied by changes at the protein level, FKBP51 protein was quantified in the Stanley Array cohort (Figure 5A), using an antibody targeting FKBP51 isoform 1, which is translated from mRNA transcript variants 1,2 and 3 . There was no significant effect of diagnosis on FKBP51 protein abundance (ANCOVA F(2, $95)=0.96, p=0.39$; Figure 5B). 

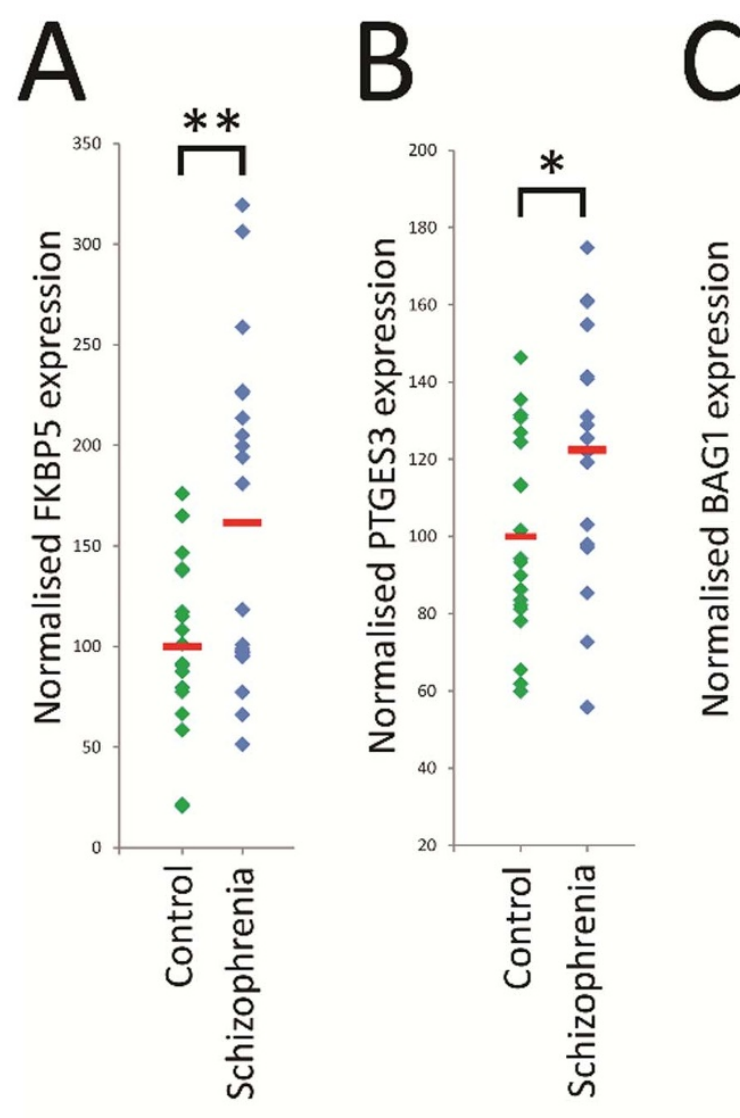

D
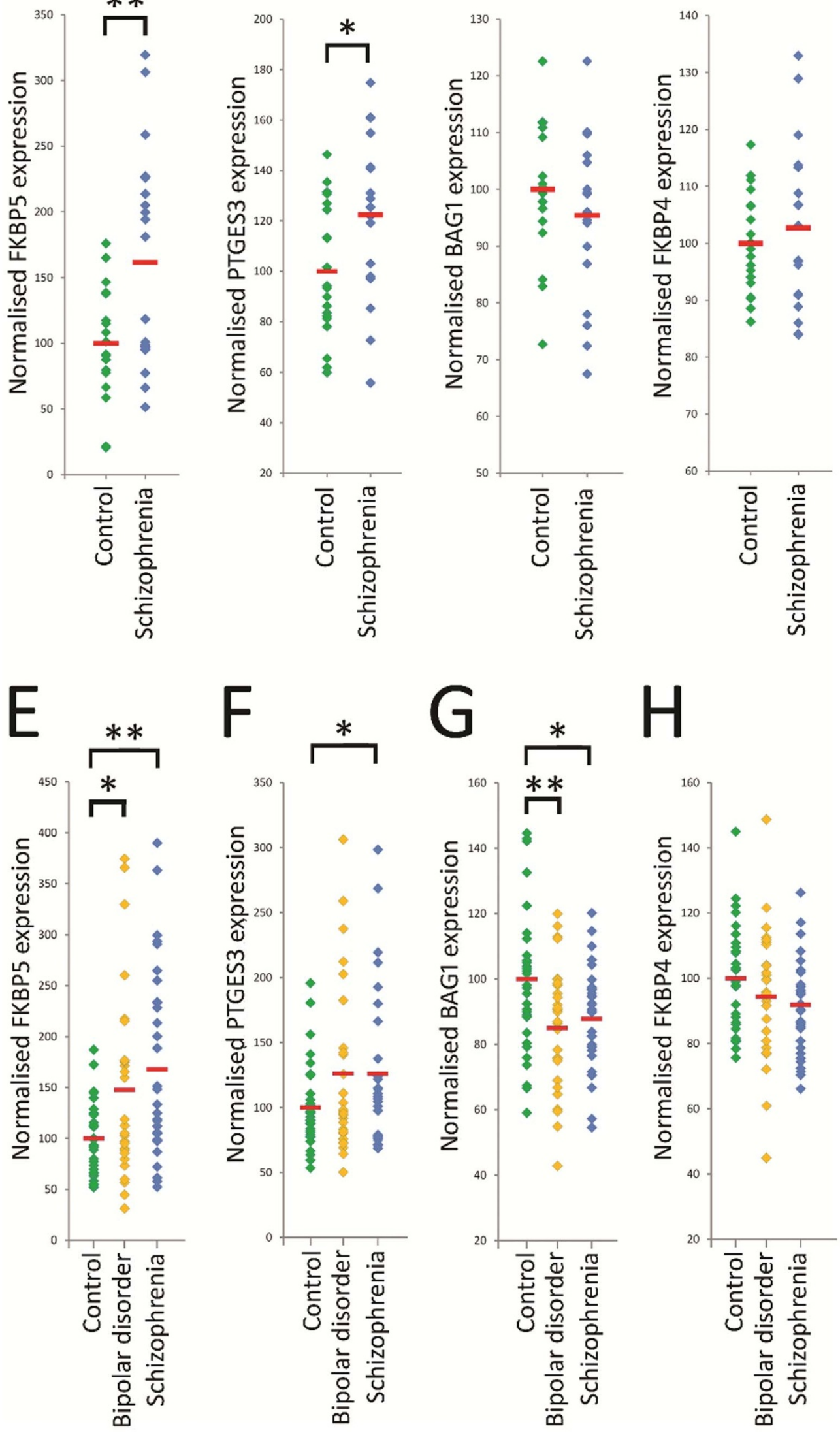

$\mathrm{H}$

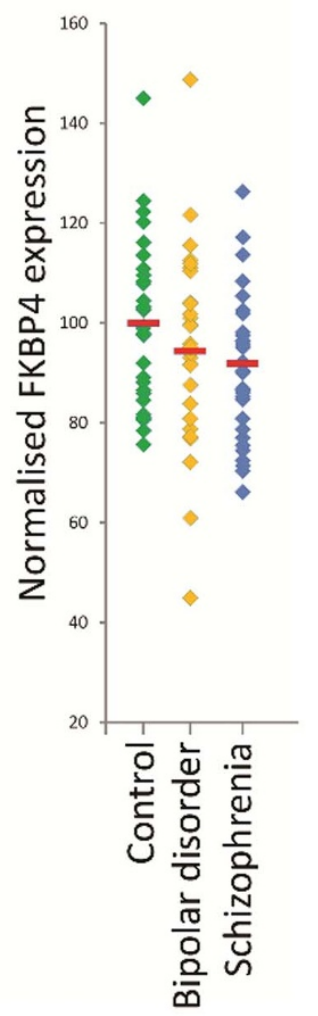

Figure 2 | Stress signaling co-factor gene expression in the DLPFC. (A-D) Normalised expression of FKBP5, PTGES3, BAG1 and FKBP4 genes in schizophrenia cases $(n=20)$ and controls $(n=20)$ from the Sydney TRC cohort; $(E-G)$ Normalised expression of FKBP5, PTGES3, BAG1 and FKBP4 in schizophrenia cases $(\mathrm{n}=35)$, bipolar disorder cases $(\mathrm{n}=31)$ and controls $(\mathrm{n}=34)$ from the Stanley Array cohort. $* p<0.05,{ }^{* *} \mathrm{p}<0.005$. 
Table 2 | Demographics of schizophrenia, bipolar disorder and matched control subjects in the Stanley Array post-mortem brain tissue cohort used in this study

\begin{tabular}{|c|c|c|c|}
\hline Stanley Array cohort & Control group $(n=35)$ & Schizophrenia group ( $\mathrm{n}=35$ ) & Bipolar disorder group $(\mathrm{n}=34)$ \\
\hline Diagnostic subtype & - & $\begin{array}{l}\text { SZ(disorg.) = } 1 \text { SZ(paranoid) }=8 \\
\text { SZ(undiff.) }=26\end{array}$ & $\begin{aligned} \mathrm{BP} 1= & 27, \mathrm{BP} 2=4, \mathrm{BPNOS}=2, \\
& \text { schizoaffective }=1\end{aligned}$ \\
\hline Age in years (range) & $44.2(31-60)$ & $42.6(19-59)$ & $45.4(19-64)$ \\
\hline & $9 F, 26$ & $9 \mathrm{~F}$, & 18 \\
\hline here & $16 \mathrm{~L}, 19 \mathrm{R}$ & $17 \mathrm{~L}, 18 \mathrm{R}$ & $19 \mathrm{~L}, 15 \mathrm{R}$ \\
\hline & $6.61+/-0.27$ & $6.48+/-0.24$ & $6.43+/-0.30$ \\
\hline$s) \pm S D$ & $29.4+/-12.9$ & $31.4+/-15.5$ & $37.9+/-18.6$ \\
\hline & $7.23+/-0.87$ & $7.36+/-0.61$ & $7.34+/-0.88$ \\
\hline Manner of death & $\begin{array}{l}\text { natural }=35 \text { (heart } \\
\text { attack }=31, \text { other }=4 \text { ) }\end{array}$ & $\begin{array}{l}\text { natural }=28, \text { suicide }=7 \text {, (heart attack } \\
=15, \text { overdose }=4, \text { pneumonia }=5, \\
\text { hanging }=3, \text { jumped }=3, \text { other }=5 \text { ) }\end{array}$ & $\begin{array}{l}\text { natural }=19, \text { suicide }=15 \text { (heart attack } \\
=7, \text { overdose }=12, \text { drowning }=3, \\
\text { asphyxiation }=2, \text { hanging }=2, \\
\text { other }=8 \text { ) }\end{array}$ \\
\hline Age ly & - & $21.319-3$ & $25.3(14-48)$ \\
\hline & & 013 & \\
\hline $\begin{array}{l}\text { Lifetime antipsychotics (Fluphenazine } \\
\text { equiv., mg) }\end{array}$ & - & $85004+/-100335$ & $10212+/-22871$ \\
\hline $\begin{array}{l}\text { Antidepressant use } \\
\text { Type of antidepressant* }\end{array}$ & yes $=0$, no $=35$ & $\begin{array}{c}\text { yes }=9, \text { no }=26 \\
\text { SSRI }=4 \text { SNRI }=0 \text { SARI }=2, \\
\text { TCA }=2, \text { other }=2\end{array}$ & $\begin{array}{c}\text { yes }=19, \text { no }=15 \\
\mathrm{SSRI}=9 \mathrm{SNRI}=4 \mathrm{SARI}=5 \mathrm{TCA}=6, \\
\text { other }=1\end{array}$ \\
\hline
\end{tabular}

Abbreviations: M- male, L- left, R- right, SD- standard deviation, PMI- post-mortem interval, RIN- RNA integrity number, y-years, BP1-bipolar disorder type I, BP2- bipolar disorder type II, BPNOS- bipolar disorder not otherwise specified, SZ- schizophrenia. Data quoted are mean (range) +/- standard deviation. *Note- some individuals took multiple antidepressant medications.

Effects of FKBP5 genotype on FKBP5 mRNA and FKBP51 protein expression in the DLPFC. Individuals in the Stanley Array cohort were genotyped at 8 SNPs within the FKBP5 gene (Figure 5C). A significant main effect of genotype on FKBP5 mRNA levels was observed for rs4713916 (ANOVA $\mathrm{F}(2,76)=3.49, p<0.05$ ) within combined control, bipolar disorder and schizophrenia samples. FKBP5 mRNA expression was $27.5 \%$ lower in TT homozygotes ( $\mathrm{n}$ $=9)$ than TC heterozygotes $(n=32, p<0.05)$. There were no other significant main effects of genotyped SNPs on FKBP5 gene expression (all $\mathrm{F}<2.23, p>0.11$ ), nor were there significant genotype $\mathrm{x}$ diagnosis interactions. There were also no significant main effects of genotype on FKBP51 protein levels in the DLPFC (all $\mathrm{F}<1.77, p>0.18$ ).

\section{Discussion}

We have identified, for the first time, alterations in FKBP5 transcriptional regulation in the brain in schizophrenia and bipolar disorder, consistent with accumulating evidence for a role of the FKBP5 gene in the etiology of psychiatric illness ${ }^{23,24,26,28}$. We also provide evidence for the involvement of two other GR co-factors, PTGES3 and BAG1, in psychotic illness. Considering these findings in the context of our previous work with the Stanley Array schizophrenia/bipolar disorder cohort, it is likely that altered expression of FKBP5, PTGES3 and BAG1 mRNAs does not occur independently, but occurs in parallel with decreased expression of multiple GR mRNA transcripts ${ }^{16}$ and increased expression of the functional, truncated GR $\alpha$-D1 protein isoform ${ }^{35}$. Taken together, our results suggest widespread stress signaling alterations in both schizophrenia and bipolar illness.

We did not replicate the previous report of increased HSPA1A, HSPA1B and HSPB1 expression in the DLPFC found in 14 patients with schizophrenia relative to controls ${ }^{34}$. This may be due to the large variance in mRNA observed for these transcripts perhaps due to increased biological heterogeneity observed in schizophrenia, or differences in the sensitivities and target specificities of microarray, RNA-Seq and qPCR methods. Diagnostic differences were restricted to co-factors (FKBP5, PTGES3, BAG1) whose functions more specifically relate to steroid hormone signalling, and were not seen in HSP chaperones which have a diverse range of additional functions.

FKBP51 regulates stress responsiveness by inhibiting GR nuclear translocation (Figure 1) ${ }^{17}$. However, given that increases in FKBP51 protein were not observed in schizophrenia and bipolar disorder, it is somewhat unclear what functional impact mRNA alterations may have in the DLPFC. In this study, RNA-Seq mRNA alterations arose from both FKBP5 mRNA variants 1-3 collectively, and FKBP5 mRNA variant 4 (see Supplementary Table S2), while qPCR changes were detected using a pan FKBP5 mRNA probe. At the protein level, we measured FKBP51 isoform 1, which is translated from FKBP5 mRNA transcripts 1-3, but not the truncated FKBP51 isoform 2, which is translated from FKBP5 mRNA transcript 4. Therefore, it is possible that we failed to detect diagnostic differences in FKBP51 isoform 2, which arose from dysregulation of FKBP5 mRNA variant 4 expression. For FKBP51 isoform 1, since we measured steady-state protein levels (one snap-shot in time), and this molecule is involved in many active cellular process, it is possible that a large increase in FKBP5 mRNA would result in more FKBP51 protein that is then readily used and degraded, resulting in no apparent change in overall protein levels. Further studies are needed to clarify if FKBP5 turnover, subcellular localization or binding to GR is changed in people with psychotic mental illness. A discordance of FKBP5 mRNA and protein findings has also previously reported in the context of genotype effects in healthy individuals ${ }^{23}$. The extent of FKBP5 mRNA upregulation in psychotic illness observed in this study, and its occurrence in two independent post-mortem cohorts, suggest that FKBP5 mRNA over-expression may be a robust feature of these illnesses.

It is possible that FKBP5 mRNA over-expression in the DLPFC may occur in schizophrenia and bipolar disorder cases due to hypercortisolemia, which has been described in these illnesses ${ }^{10,11}$. FKBP5 gene expression is upregulated by glucocorticoids through GR in rodent and primate brain ${ }^{36-40}$, and in squirrel monkeys, chronic hypercortisolemia is compensated for by over-expression of FKBP $5^{41}$. Therefore, it is possible that FKBP5 upregulation may have arisen in an attempt to 'buffer' chronically increased stress/cortisol in individuals in this cohort. Consistent with this hypothesis, FKBP5 mRNA was negatively correlated with GR mRNA, which is known to be downregulated by hypercortisolemia ${ }^{42}$. Unfortunately, data on circulating cortisol levels for individuals in these cohorts are not available. Alternatively, 'high-induction' FKBP5 polymorphisms may contribute to FKBP5 mRNA upregulation in psychiatric illness. The rs1360780 FKBP5 polymorphism has been shown to influence 


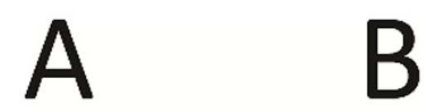

C

D
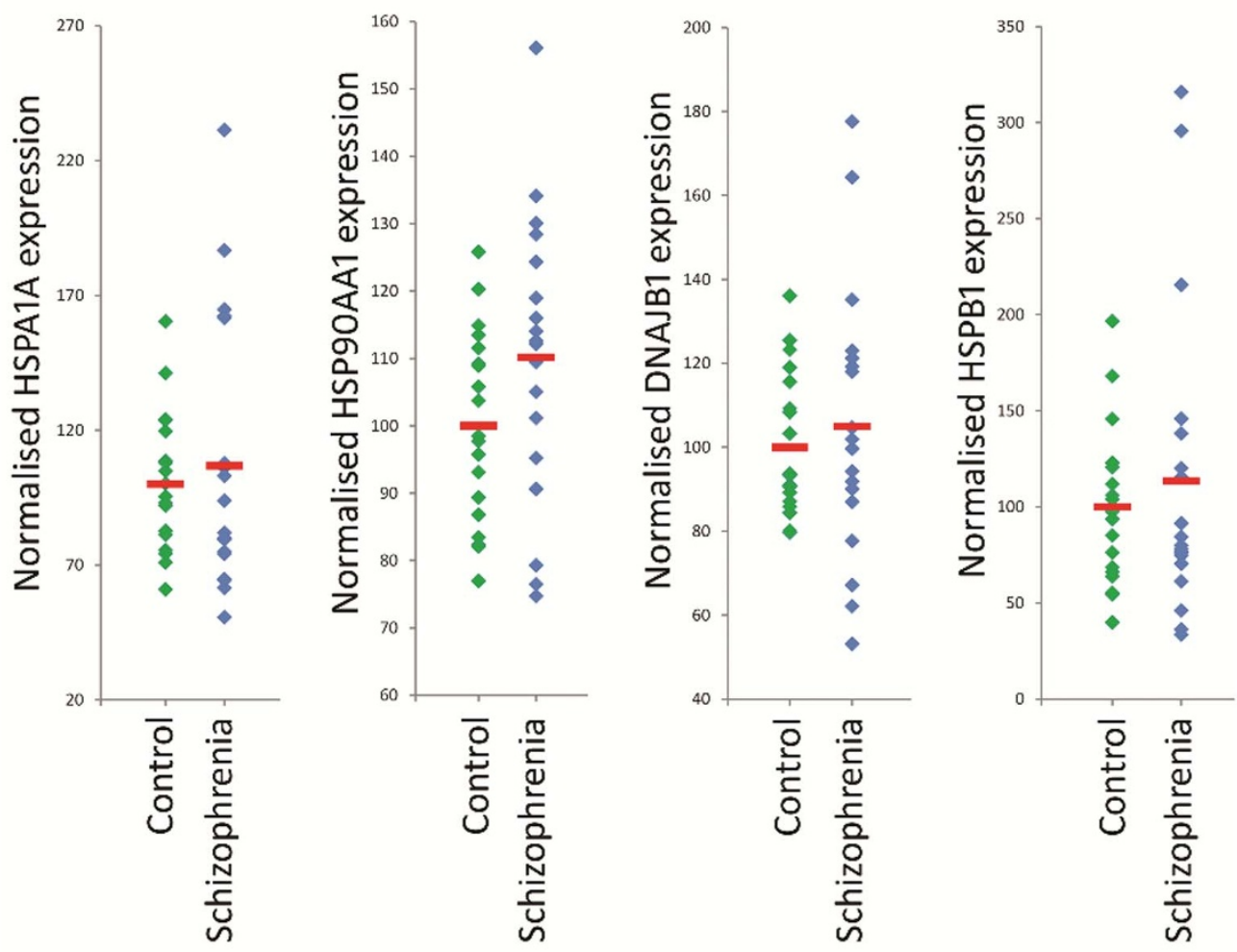

$E$

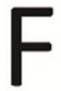

G

$\mathrm{H}$
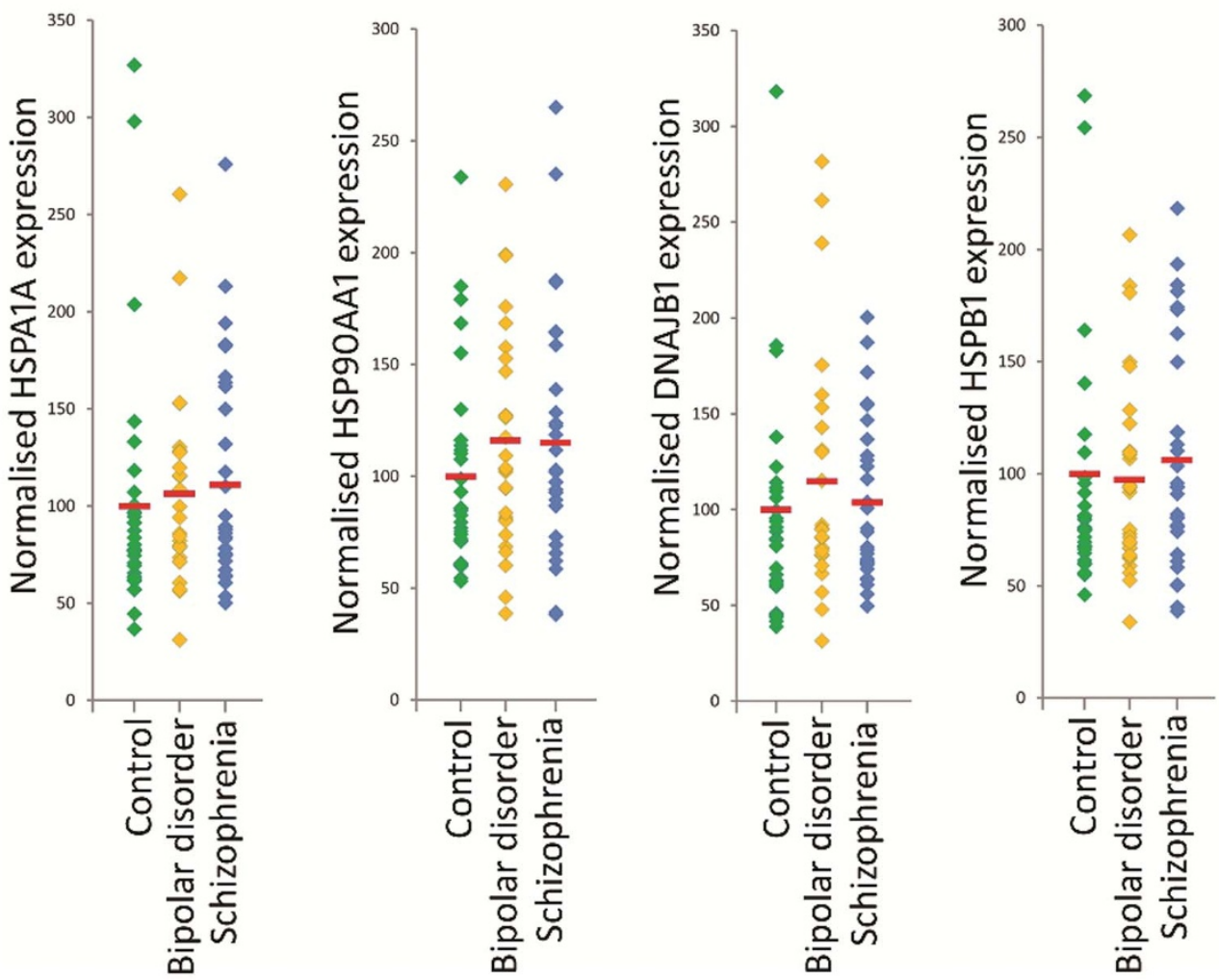

Figure $3 \mid$ mRNA expression of HSP chaperones in the DLPFC. (A-D) Normalised expression of HSPA1A, HSP90AA1, DNAJB1 and HSPB1 genes in schizophrenia cases $(\mathrm{n}=20)$ and controls $(\mathrm{n}=20)$ from the Sydney TRC cohort; $(\mathrm{E}-\mathrm{G})$ Normalised expression of HSPA1A, HSP90AAl, DNAJB1 and HSPB1 in schizophrenia cases $(\mathrm{n}=35)$, bipolar disorder cases $(\mathrm{n}=31)$ and controls $(\mathrm{n}=34)$ from the Stanley Array cohort. 


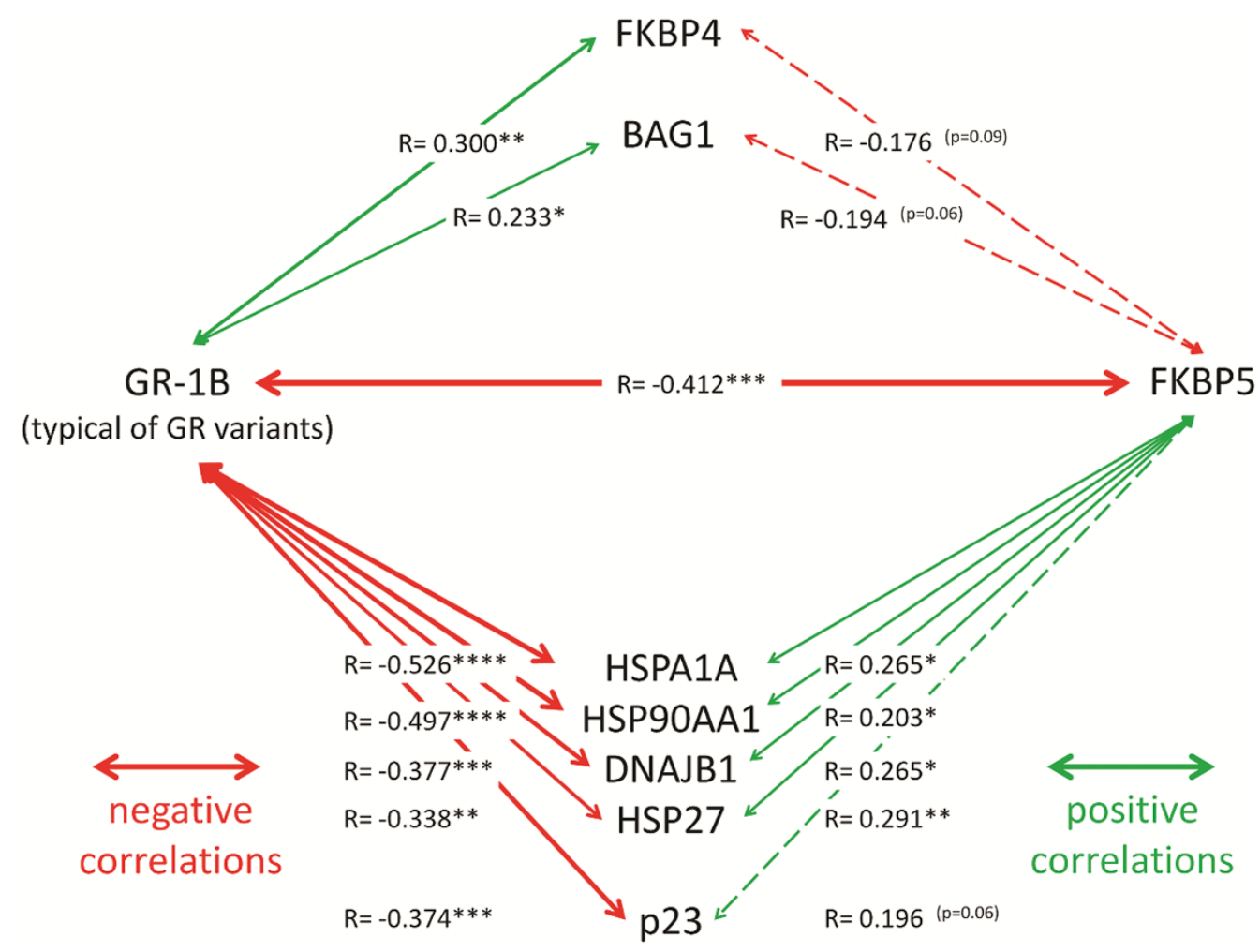

Figure 4 Correlations between mRNA expression levels of GR-1B, stress signaling co-factors and chaperones in the DLPFC. Red arrows represent negative correlations, and green arrows positive correlations, in the Stanley Array cohort. While correlations of chaperones/co-factors with GR-1B mRNA are illustrated, very similar correlations with total GR mRNA, GR-1C and GR-1H were seen. ${ }^{*} p=0.05,{ }^{* *} p<0.005,{ }^{* * *} p<0.0005$, $* * * * p<0.00005$.

FKBP5 gene transcription in vitro ${ }^{26}$, and FKBP5 mRNA/FKBP51 protein expression in blood lymphocytes ${ }^{23}$. Neither this SNP, nor the seven others genotyped, were over-represented in schizophrenia and bipolar disorder cases in this study, and therefore are not likely to contribute to the observed diagnostic mRNA differences. Albeit with small sample size for genetic association, our data do provide some supporting evidence for a biological effect of FKBP5 polymorphisms in human frontal cortex, since we found that the rs4713916 TT genotype was associated with reduced FKBP5 mRNA levels in the DLPFC. This finding supports previous indications that the TT genotype may be functional, as it is linked with impaired recovery from social stress ${ }^{43}$. How impaired stress recovery may relate mechanistically to decreased prefrontal FKBP5 mRNA expression, as observed in TT homozygotes in this study, remains to be determined.

P23, encoded by the gene PTGES3, also plays a key role in glucocorticoid signaling and was increased at the mRNA level in the DLPFC in individuals with schizophrenia in our study. In the GR heterocomplex in vitro, p23 is an obligatory co-factor ${ }^{19}$ and is the limiting component of the complex ${ }^{44}$, functioning to stabilize the interaction of the complex with GR in the cytoplasm. Paradoxically, p23 also acts in the nucleus to inhibit GR-mediated gene transcription $^{45}$ and disassemble GR transcriptional machinery in the nucleus ${ }^{46}$. In vivo, p23 is critical for appropriate glucocorticoid responsiveness ${ }^{47}$. As a result, any increase in p23 protein synthesis, abundance and/or function in schizophrenia, which could occur due to increased PTGES3 mRNA expression, could profoundly impact cellular stress signaling.

Downregulation of BAG1 gene expression in schizophrenia and bipolar disorder is consistent with other recent work that also implicates Bag1 in the pathophysiology of affective illness. A significant decrease of small magnitude (12.1\%) was observed in the Stanley Array cohort, but not in the TRC cohort, possibly due to the smaller TRC cohort sample size, or neurobiological differences between indi- viduals in the two cohorts. BAG1 impairs GR folding by interacting with $\mathrm{Hsp}_{70}{ }^{48}$, facilitates complex degradation ${ }^{49}$ and inhibits GRmediated target gene transactivation ${ }^{50,51}$. Upregulation of BAG1 due to chronic stress has been observed selectively in female rats ${ }^{52}$. Mood stabilizer treatments for bipolar disorder have been shown to upregulate BAG1 expression ${ }^{30}$, while BAG1 downregulation may impair recovery from stress-induced depression-like behaviors ${ }^{53}$. Although BAG1 mRNA did not vary according to antidepressant or antipsychotic exposure in this study, it is possible that decreased BAG1 expression may be linked to affective disturbances that can occur in both schizophrenia and bipolar disorder.

Strong correlations between mRNA expression of GR, co-factors and chaperones were evident in this study. Counter-intuitively, the mRNA expression of GR and 4/5 of its obligatory co-factors/chaperones (PTGES3, DNAJB1, HSPA1A and HSP90AA1) were inversely correlated. It is plausible that these co-factors and chaperones are coregulated by a common mechanism, whose function is to normalize stress signaling by compensating for changes in GR expression, creating homeostasis. Interestingly, the expression of two co-factors, FKBP5 and FKBP4, which inhibit and facilitate GR nuclear translocation respectively, were also negatively correlated, suggesting that these co-factors are not coordinatedly up-regulated to 'balance' their effects, but may be reciprocally regulated to exaggerate their individual effects.

In this study, we have identified dysregulation of stress signaling cofactors FKBP5, PTGES3 and BAG1 in the DLPFC of bipolar disorder and/or schizophrenia patients, within a network of transcripts (consisting of GR, HSP chaperones and cofactors) that were highly correlated. We hypothesize that the coordinated regulation of stress signaling transcripts is integral to appropriate stress responsiveness in the frontal cortex, and that this process may be disrupted in those with psychiatric illness. Future work investigating GR, FKBP5, PTGES3 and BAG1 in schizophrenia and bipolar disorder may identify mechanisms underlying failed adaptation to stress in schizo- 
A

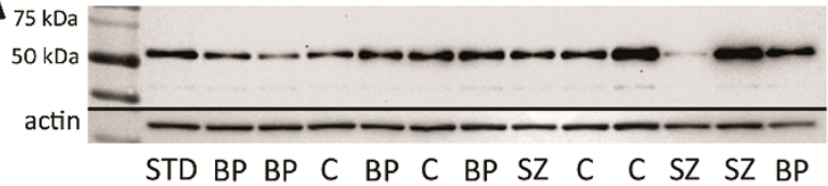

C

\begin{tabular}{|c|c|c|c|c|c|}
\hline $\begin{array}{l}\text { dbSNP } \\
\text { rs \# }\end{array}$ & $\begin{array}{l}\text { Location } \\
\text { (UCSC build } \\
\text { Hg19, Feb } \\
\text { 2009) }\end{array}$ & $\begin{array}{l}\text { position of gene on } \\
\text { chr. } 6 \text {, relative to } \\
\text { FKBP5 } \\
\text { transcript variant } 1\end{array}$ & $\begin{array}{l}\text { SNP } \\
\text { (major/ } \\
\text { minor } \\
\text { allele) }\end{array}$ & MAF & $\begin{array}{l}\text { HWE } \\
p- \\
\text { value }\end{array}$ \\
\hline rs4713916 & 35669983 & $\begin{array}{l}\text { promoter region } \\
\text { (upstream of exon 1) }\end{array}$ & $\mathrm{C} / \mathrm{T}$ & 0.306 & 0.388 \\
\hline rs9470080 & 35646435 & intron 1 & $\mathrm{G} / \mathrm{A}$ & 0.350 & 0.442 \\
\hline rs4713902 & 35614026 & intron 1 & $\mathrm{~A} / \mathrm{G}$ & 0.237 & 0.430 \\
\hline rs 1360780 & 35607571 & intron 2 & $\mathrm{G} / \mathrm{A}$ & 0.330 & 0.616 \\
\hline rs9296158 & 35567082 & intron 5 & $\mathrm{C} / \mathrm{T}$ & 0.330 & 0.616 \\
\hline rs 7757037 & 35548236 & intron 8 & $\mathrm{C} / \mathrm{T}$ & 0.445 & 0.465 \\
\hline rs 3800373 & 35542476 & 3'UTR & $\mathrm{T} / \mathrm{G}$ & 0.305 & 0.549 \\
\hline rs 1043805 & 35541432 & 3' UTR & $\mathrm{T} / \mathrm{A}$ & 0.177 & 0.949 \\
\hline
\end{tabular}

$B$

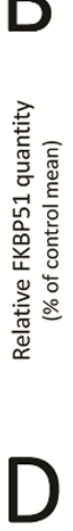

D

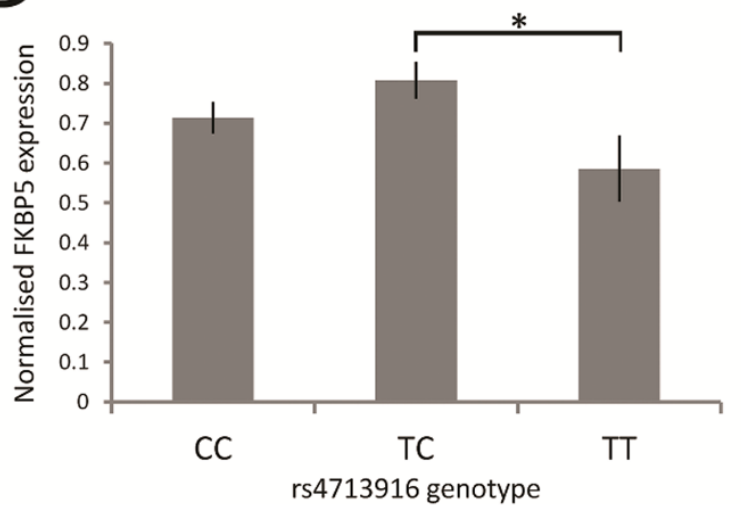

Figure 5 | FKBP51 protein expression and FKBP5 genotype effects in the Stanley Array cohort. (A) representative FKBP51 western blot; (B) FKBP51 protein abundance in schizophrenia, bipolar disorder and control groups; (C) Details of genotyped FKBP5 SNPs. For each SNP, the bases occurring on the minus strand are described; (D) FKBP5 mRNA levels in the DLPFC according to rs4713916 genotype, in CC homozygotes ( $\mathrm{n}=44$ ), TC heterozygotes $(\mathrm{n}=32)$ and TT homozygotes $(\mathrm{n}=9)$. Abbreviations: C- control, BP- bipolar disorder, SZ- schizophrenia, kDa- kilodaltons, MAF- minor allele frequency, HWE- Hardy-Weinberg Equilibrium.

phrenia and bipolar disorder, and pave the way for strategies to better buffer the effects of stress in individuals vulnerable to poor stress responses.

\section{Methods}

Tissue collection. This study was carried out in accordance with the declaration of Helsinki, after approval by the Human Research Ethics Committee at the University of NSW (\#HREC07261). Written informed consent for use of tissues in the study was obtained from next of kin.

RNA sequencing study. Tissue from the DLPFC (BA46) of 20 controls and 20 individuals with schizophrenia (the Sydney TRC cohort) was obtained from the New South Wales Tissue Resource Centre (Table 1) ${ }^{54}$. The RNA-Seq performed in this study, employing the SOLiD system (Life Technologies, Carlsbad, CA) has been previously described in detail ${ }^{55}$. On average, $\sim 50 \%$ of the $135,355,990$ unpaired reads per individual, each 50 nucleotides in length, were mapped to the genome, consistent with other studies ${ }^{56}$. There were no diagnostic differences in read length or average read count. The mapped reads were aggregated into 33,105 transcripts. For each individual sample, an aggregate expression value for each co-factor and chaperone gene was calculated by taking the geometric mean of the number of mapped reads (normalised for total read length and total number of sequencing reads) for all known transcripts of that gene. Further information is available as Supplementary Methods.

Quantitative real-time PCR, western blotting and genotyping study. DLPFC tissues (middle frontal gyrus) from the Stanley Array collection cohort were used for qPCR, western blotting and genotyping (Table 2). Genomic DNA (from 34 schizophrenia/30 bipolar disorder/31 control), total mRNA (from 35 schizophrenia/ 31 bipolar disorder/34 control), and crude protein homogenate (from 35 schizophrenia/34 bipolar disorder/35 control) were provided by the Stanley Medical Research Institute, having been extracted as previously described ${ }^{15}$.

Pre-designed Taqman Gene Expression Assays (Applied Biosystems, Foster City, CA, USA) were chosen for each of the genes analysed: FKBP5 (Hs01561006_m1), FKBP4 (Hs00427038_g1), PTGES3 (Hs00832847_gH), BAG1 (Hs00185390_m1), HSPA1A (Hs00271229_s1), HSP90AA1 (Hs00743767_sH), DNAJB1 (Hs00428680_ml) and HSPB1 (Hs00356629_g1). For FKBP5, the Taqman primer/probe set detected all FKBP5 mRNA transcript variants (1-4), at the exon 5-6 junction (variants 1, 3 and 4) or exon 6-7 junction (variant 2). All reactions were performed in triplicate. Expression levels were normalized to the geometric mean of four validated 'housekeeper' genes, from distinct biological pathways, whose raw expression levels did not differ between diagnostic groups: ACTB (Hs99999903_m1), B2M (Hs99999907_m1), TBP (Hs00427620_ml) and UBC (Hs00824723_ml).

Western blotting was conducted and data normalized as previously described ${ }^{14}$, using the ab2901 anti-FKBP51 primary antibody (Abcam, Cambridge, UK), which targets amino acids 395-407 of FKBP51 isoform 1 (translated from FKBP5 transcript variants 1-3). Samples were run in duplicate in separate experimental runs, and the geometric mean of both runs calculated. Further information is available as Supplementary Methods.

Eight promoter or putative functional FKBP5 gene SNPs were genotyped (Figure 5) at the Australian Genome Research Facility using Sequenom MassArray method previously described ${ }^{16}$, with an assay pass rate of $96-98 \%$. PLINK software (version 1.06, http://pngu.mgh.harvard.edu/purcell/plink) ${ }^{57}$ was used for HardyWeinberg equilibrium testing.

Prior to analysis, all data were either approximately normally distributed, or log transformed to yield an approximately normal distribution (skewness between -1 and 1). Population outliers, whose values were greater than 2 standard deviations from the diagnostic group mean, were excluded from all analyses. For all mRNA measures, Pearson correlation analyses were conducted with age, brain $\mathrm{pH}, \mathrm{PMI}$ and RIN for all individuals. Within schizophrenia and bipolar disorder cases, correlation analyses were also conducted with age-of-onset, duration-of-illness and antipsychotic drug measures (ascertained by converting all antipsychotic drugs taken to lifetime chlorpromazine [TRC cohort] or fluphenazine [Stanley Array cohort] equivalents). For all mRNA transcripts, analyses of covariance (ANCOVA) were used with age, $\mathrm{pH}$ and RIN as covariates, to identify effects of diagnosis and gender. For analysis of suicide and antidepressant use, schizophrenia and bipolar disorder groups were subdivided according to suicide and antidepressant status) and ANCOVA analysis performed. For FKBP51 protein, ANCOVA was used with age, $\mathrm{pH}$ and PMI as covariates. The observed power of all ANCOVA analyses is given in Supplementary Table S1. Planned comparisons of schizophrenia, bipolar disorder and control groups were made using Fisher's LSD post-hoc test. Two-way ANOVAs were also used, with diagnosis and genotype as independent factors, to identify the effects of genotype on mRNA expression. To analyze correlations between GR transcripts, co-factors and chaperones, Spearman's correlations with non-transformed data were used. Statistica software (Statsoft, Oklahoma, USA) was used for all analyses. 
1. Walker, E. F. \& Diforio, D. Schizophrenia: A Neural Diathesis-Stress Model. Psychol Rev 104, 667-685 (1997).

2. Arseneault, L. et al. Childhood trauma and children's emerging psychotic symptoms: A genetically sensitive longitudinal cohort study. Am J Psych 168, 65-72 (2011).

3. Khashan, A. S. et al. Higher risk of offspring schizophrenia following antenatal maternal exposure to severe adverse life events. Arch Gen Psychiatry 65, 146-152 (2008).

4. Agid, O. et al. Environment and vulnerability to major psychiatric illness: A case control study of early parental loss in major depression, bipolar disorder and schizophrenia. Mol Psychiatry 4, 163-172 (1999).

5. Yung, A. R. et al. Mapping the onset of psychosis: The Comprehensive Assessment of At-Risk Mental States. Aust NZ J Psychiatry 39, 964-971 (2005)

6. Day, R. et al. Stressful life events preceding the acute onset of schizophrenia: A cross-national study from the World Health Organization. Cult Med Psychiatr 11, 123-205 (1987).

7. Read, J., Agar, K., Argyle, N. \& Aderhold, V. Sexual and physical abuse during childhood and adulthood as predictors of hallucinations, delusions and thought disorder. Psychol Psychother 76, 1-22 (2003).

8. Read, J., van Os, J., Morrison, A. P. \& Ross, C. A. Childhood trauma, psychosis and schizophrenia: a literature review with theoretical and clinical implications. Acta Psychiatr Scand 112, 330-350 (2005).

9. Gallagher, P., Watson, S., Smith, M. S., Young, A. H. \& Ferrier, I. N. Plasma cortisol-dehydroepiandrosterone (DHEA) ratios in schizophrenia and bipolar disorder. Schizophr Res 90, 258-265 (2007).

10. Watson, S., Gallagher, P., Ritchie, J. C., Ferrier, I. N. \& Young, A. H. Hypothalamic-pituitary-adrenal axis function in patients with bipolar disorder. Br J Psychiatry 184, 496-502 (2004).

11. Bradley, A. J. \& Dinan, T. G. A systematic review of hypothalamic-pituitaryadrenal axis function in schizophrenia: implications for mortality. J Psychopharmacol 24, 91-118 (2010).

12. Perlman, W. R., Webster, M. J., Kleinman, J. E. \& Weickert, C. S. Reduced glucocorticoid and estrogen receptor alpha messenger ribonucleic acid levels in the amygdala of patients with major mental illness. Biol Psychiatry 56, 844-852 (2004)

13. Webster, M. J., Knable, M. B., O’Grady, J., Orthmann, J. \& Weickert, C. S. Regional specificity of brain glucocorticoid receptor mRNA alterations in subjects with schizophrenia and mood disorders. Mol Psychiatry 7, 985-994 (2002).

14. Sinclair, D., Tsai, S. Y., Woon, H. G. \& Shannon Weickert, C. Abnormal glucocorticoid receptor mRNA and protein isoform expression in the prefrontal cortex in psychiatric illness. Neuropsychopharmacol 36, 2698-2709 (2011)

15. Sinclair, D., Webster, M. J., Fullerton, J. M. \& Shannon Weickert, C. Glucocorticoid receptor mRNA and protein isoform alterations in the orbitofrontal cortex in schizophrenia and bipolar disorder. BMC Psychiatry 12, 84, doi:10.1186/1471-244X-12-84 (2012)

16. Sinclair, D., Fullerton, J. M., Webster, M. J. \& Shannon Weickert, C. Glucocorticoid receptor $1 \mathrm{~B}$ and $1 \mathrm{C}$ mRNA transcript alterations in schizophrenia and bipolar disorder, and their possible regulation by GR gene variants. PLoS One 7, e31720, doi:10.1371/journal.pone.0031720 (2012).

17. Grad, I. \& Picard, D. The glucocorticoid responses are shaped by molecular chaperones. Mol Cell Endocrinol 275, 2-12 (2007).

18. Dittmar, K. D., Banach, M., Galigniana, M. D. \& Pratt, W. B. The role of DnaJ-like proteins in glucocorticoid receptor.hsp 90 heterocomplex assembly by the reconstituted hsp90.p60.hsp70 foldosome complex. J Biol Chem 273, 7358-7366 (1998)

19. Dittmar, K. D., Demady, D. R., Stancato, L. F., Krishna, P. \& Pratt, W. B. Folding of the glucocorticoid receptor by the heat shock protein (hsp) 90-based chaperone machinery. The role of $\mathrm{p} 23$ is to stabilize receptor.hsp 90 heterocomplexes formed by hsp90.p60.hsp70. J Biol Chem 272, 21213-21220 (1997).

20. Dittmar, K. D., Hutchison, K. A., Owens-Grillo, J. K. \& Pratt, W. B. Reconstitution of the steroid receptor.hsp90 heterocomplex assembly system of rabbit reticulocyte lysate. J Biol Chem 271, 12833-12839 (1996).

21. Dittmar, K. D. \& Pratt, W. B. Folding of the glucocorticoid receptor by the reconstituted Hsp90-based chaperone machinery. The initial hsp90.p60.hsp70dependent step is sufficient for creating the steroid binding conformation. J Biol Chem 272, 13047-13054 (1997).

22. Willour, V. L. et al. Family-based association of FKBP5 in bipolar disorder. Mol Psychiatry 14, 261-268 (2009).

23. Binder, E. B. et al. Polymorphisms in FKBP5 are associated with increased recurrence of depressive episodes and rapid response to antidepressant treatment. Nat Genet 36, 1319-1325 (2004).

24. Binder, E. B. et al. Association of FKBP5 polymorphisms and childhood abuse with risk of posttraumatic stress disorder symptoms in adults. JAMA 299, 1291-1305 (2008)

25. Binder, E. B. The role of FKBP5, a co-chaperone of the glucocorticoid receptor in the pathogenesis and therapy of affective and anxiety disorders. Psychoneuroendocrinology 34 Suppl 1, S186-195 (2009).

26. Klengel, T. et al. Allele-specific FKBP5 DNA demethylation mediates genechildhood trauma interactions. Nat Neurosci 16, 33-41 (2013).

27. Simons, C. J., van Winkel, R. \& Group. Intermediate phenotype analysis of patients, unaffected siblings, and healthy controls identifies VMAT2 as a candidate gene for psychotic disorder and neurocognition. Schizophr Bull 39 848-856, doi:10.1093/schbul/sbs067 (2013).

28. Tatro, E. T. et al. Differential expression of immunophilins FKBP51 and FKBP52 in the frontal cortex of HIV-infected patients with major depressive disorder. J Neuroimmune Pharmacol 4, 218-226 (2009).

29. Perez-Ortiz, J. M., Garcia-Gutierrez, M. S., Navarrete, F., Giner, S. \& Manzanares, J. Gene and protein alterations of FKBP5 and glucocorticoid receptor in the amygdala of suicide victims. Psychoneuroendocrinology (2012).

30. Zhou, R. et al. The anti-apoptotic, glucocorticoid receptor cochaperone protein BAG-1 is a long-term target for the actions of mood stabilizers. J Neurosci $\mathbf{2 5}$, 4493-4502 (2005).

31. Kim, J. J. et al. Association analysis of heat shock protein 70 gene polymorphisms in schizophrenia. Eur Arch Psychiatry Clin Neurosci 258, 239-244 (2008).

32. Pae, C. U. et al. The impact of heat shock protein 70 gene variations on clinical presentation and outcome in schizophrenic inpatients. Neuropsychobiology 59, 135-141 (2009).

33. Kakiuchi, C. et al. Association analysis of HSP90B1 with bipolar disorder. J Hum Genet 52, 794-803 (2007).

34. Arion, D., Unger, T., Lewis, D. A., Levitt, P. \& Mirnics, K. Molecular Evidence for Increased Expression of Genes Related to Immune and Chaperone Function in the Prefrontal Cortex in Schizophrenia. Biol Psychiatry 62, 711-721 (2007).

35. Sinclair, D., Tsai, S. Y., Woon, H. G. \& Weickert, C. S. Abnormal glucocorticoid receptor mRNA and protein isoform expression in the prefrontal cortex in psychiatric illness. Neuropsychopharmacol 36, 2698-2709 (2011).

36. Lee, R. S. et al. Chronic corticosterone exposure increases expression and decreases deoxyribonucleic acid methylation of Fkbp5 in mice. Endocrinology 151, 4332-4343 (2010).

37. Scharf, S. H., Liebl, C., Binder, E. B., Schmidt, M. V. \& Muller, M. B. Expression and regulation of the Fkbp5 gene in the adult mouse brain. PLoS One 6, e16883, doi:10.1371/journal.pone.0016883 (2011).

38. Wagner, K. V. et al. Differences in FKBP51 regulation following chronic social defeat stress correlate with individual stress sensitivity: influence of paroxetine treatment. Neuropsychopharmacol 37, 2797-2808 (2012)

39. Guidotti, G. et al. Glucocorticoid Receptor and FKBP5 Expression Is Altered Following Exposure to Chronic Stress: Modulation by Antidepressant Treatment. Neuropsychopharmacol 38, 616-627 (2013).

40. Hubler, T. R. \& Scammell, J. G. Intronic hormone response elements mediate regulation of FKBP5 by progestins and glucocorticoids. Cell Stress Chaperones $\mathbf{9}$, 243-252 (2004)

41. Westberry, J. M., Sadosky, P. W., Hubler, T. R., Gross, K. L. \& Scammell, J. G. Glucocorticoid resistance in squirrel monkeys results from a combination of a transcriptionally incompetent glucocorticoid receptor and overexpression of the glucocorticoid receptor co-chaperone FKBP51. J Steroid Biochem Mol Biol 100 34-41 (2006)

42. Patel, P. D., Katz, M., Karssen, A. M. \& Lyons, D. M. Stress-induced changes in corticosteroid receptor expression in primate hippocampus and prefrontal cortex. Psychoneuroendocrinology 33, 360-367 (2008).

43. Ising, M. et al. Polymorphisms in the FKBP5 gene region modulate recovery from psychosocial stress in healthy controls. Eur J Neurosci 28, 389-398 (2008).

44. Morishima, Y. et al. The hsp90 cochaperone p23 is the limiting component of the multiprotein hsp90/hsp70-based chaperone system in vivo where it acts to stabilize the client protein: hsp90 complex. J Biol Chem 278, 48754-48763 (2003).

45. Wochnik, G. M. et al. Inhibition of GR-mediated transcription by 23 requires interaction with Hsp90. FEBS Lett 560, 35-38 (2004).

46. Freeman, B. C. \& Yamamoto, K. R. Disassembly of transcriptional regulatory complexes by molecular chaperones. Science 296, 2232-2235 (2002).

47. Grad, I. et al. The Hsp90 cochaperone p23 is essential for perinatal survival. Mol Cell Biol 26, 8976-8983 (2006).

48. Bimston, D. et al. BAG-1, a negative regulator of Hsp70 chaperone activity, uncouples nucleotide hydrolysis from substrate release. EMBO J 17, 6871-6878 (1998).

49. Luders, J., Demand, J. \& Hohfeld, J. The ubiquitin-related BAG-1 provides a link between the molecular chaperones $\mathrm{Hsc} 70 / \mathrm{Hsp} 70$ and the proteasome. J Biol Chem 275, 4613-4617 (2000)

50. Kullmann, M. et al. RAP46 is a negative regulator of glucocorticoid receptor action and hormone-induced apoptosis. J Biol Chem 273, 14620-14625 (1998).

51. Schneikert, J., Hubner, S., Martin, E. \& Cato, A. C. A nuclear action of the eukaryotic cochaperone RAP46 in downregulation of glucocorticoid receptor activity. J Cell Biol 146, 929-940 (1999).

52. Bourke, C. H. et al. Glucocorticoid sensitizers Bag1 and Ppid are regulated by adolescent stress in a sex-dependent manner. Psychoneuroendocrinology 38, 84-93 (2013).

53. Maeng, S. et al. BAG1 plays a critical role in regulating recovery from both maniclike and depression-like behavioral impairments. Proc Natl Acad Sci U S A 105, 8766-8771 (2008)

54. Weickert, C. S. et al. Selection of reference gene expression in a schizophrenia brain cohort. Aust N Z J Psychiatry 44, 59-70 (2010).

55. Fillman, S. G. et al. Increased inflammatory markers identified in the dorsolateral prefrontal cortex of individuals with schizophrenia. Mol Psychiatry 18, 206-214, doi:10.1038/mp.2012.110 (2013). 
56. Tariq, M. A., Kim, H. J., Jejelowo, O. \& Pourmand, N. Whole-transcriptome RNAseq analysis from minute amount of total RNA. Nucleic Acids Res 39, e120, doi:10.1093/nar/gkr547 (2011).

57. Purcell, S. et al. PLINK: a tool set for whole-genome association and populationbased linkage analyses. Am J Hum Genet 81, 559-575 (2007).

\section{Acknowledgments}

This work was supported by the Schizophrenia Research Institute (utilising infrastructure funding from the NSW Ministry of Health and the Macquarie Group Foundation), the University of New South Wales, and Neuroscience Research Australia. Tissues were received from the Stanley Medical Research Institute. Grant support was received from the Stanley Medical Research Institute (grant \# 09R-2158). CSW is a recipient of a National Health and Medical Research Council (Australia) Senior Research Fellowship. We express our gratitude to the individuals who donated postmortem tissues used in this study, and their families.

\section{Author contributions}

C.S.W., D.S. and S.G.F. conceived and designed the study, M.J.W. developed the Stanley Array post-mortem tissue cohort, provided human neuroanatomical expertise, performed $\mathrm{RNA} /$ protein extractions and contributed to analysis of demographic data. D.S. performed RNA/protein extractions, qPCR and Western blotting experiments, S.G.F., D.S. and C.S.W. analyzed the data, D.S., S.G.F. and C.S.W. wrote the manuscript and all authors reviewed the manuscript.

\section{Additional information}

Supplementary information accompanies this paper at http://www.nature.com/ scientificreports

Competing financial interests: The authors declare no competing financial interests.

How to cite this article: Sinclair, D., Fillman, S.G., Webster, M.J. \& Weickert, C.S Dysregulation of glucocorticoid receptor co-factors FKBP5, BAG1 and PTGES3 in prefrontal cortex in psychotic illness. Sci. Rep. 3, 3539; DOI:10.1038/srep03539 (2013).

(2) This work is licensed under a Creative Commons Attribution-

NonCommercial-NoDerivs 3.0 Unported license. To view a copy of this license, visit http://creativecommons.org/licenses/by-nc-nd/3.0 